\title{
THMs formation potential under Amino acid chlorination in a pilot-scale
}

\section{water distribution system}

\author{
Feilong Dong ${ }^{1, \mathrm{a}}, \mathrm{Xu} \mathrm{Du}^{1, \mathrm{~b}}$, Xingbo Chen ${ }^{1, \mathrm{c}}$, Cong $\mathrm{Li}^{1, \mathrm{~d}, *}$ \\ ${ }^{1}$ College of Civil Engineering and Architecture, Zhejiang University, Hangzhou 310027, China \\ adongfeilong86@126.com, bdux@futureholdings.com.cn, 'cxbgzw0901@163.com, \\ dcongil@zju.edu.cn
}

Keywords: Chlorination, Trihalomethanes (THMs), Water distribution system, Potential capacity Abstract. In recent years, the most commonly used method for the tap water disinfection is chlorination. However, a lot of disinfection byproducts (DBPs) with the nature of carcinogenicity, teratogenicity, mutagenicity can be produced as by-products in the process of chlorination. Therefore, DBPs would do great harm to the city residents' health and normal life, and lead to water pollution. Thus, the research about the impacts of DBPs in water distribution system is of great value and emergency. In this paper, not only the standard working curve of several kinds of DBPs and the effects on the potential capacity for THMs in beaker experiment were investigated, but also the impacts of different pipe materials on the potential capacity for THMs by network experiments were determine.

\section{Introduction}

Given that the research showed a direct link between the formation of THMs and the reaction between natural organic matter (NOM) and free chlorine, the environmental officials and the public began to taking great concern to DBPs since the late 1970s [1].Trihalomethanes (THMs) and haloacetic acids (HAAs) are the two major groups of DBPs, which are potentially carcinogenic [2]. Among the high-priority THMs included chloroform $\left(\mathrm{CHCl}_{3}, \mathrm{CF}\right)$, chlorodibromomethane $\left(\mathrm{CHBrCl}_{2}, \mathrm{BDCM}\right)$, bromodichloromethane $\left(\mathrm{CHBr}_{2} \mathrm{Cl}, \mathrm{DBCM}\right)$ and bromoform $\left(\mathrm{CHBr}_{3}, \mathrm{BF}\right)$, bromochloro-(BCNM) received special attention because of their potential high toxicity and occurrence in some water treatment facilities [3]. Therefore, it is important to understand the relationship between the characteristics of amino acids (AA) and THMs yields.

Although some studies were performed to characterize THMs in several source waters [4], little information was focused on the characteristics of THMs in water distribution system, especially in waters with high concentration of bromide. The chief aim of this research was to compare the potential of producing THMs from different reaction conditions.

Despite of the high volatile of THMs, treated waters - distributed in closed pipes minimizes their evaporation. They can thus be continuously generated between residual chlorine and dissolved organic matter during the water's distribution [5]. Up to now, THMs have become one of the most widely studied DBPs. The methods of THMs detection include static headspace (HS) liquid-phase microextraction, gas chromatography-mass spectrometry (GC-MS) [6], dispersive liquid-liquid microextraction and gas chromatography (GC) with electro capture detection (ECD) [7]. In this work, dispersive liquid-liquid microextraction and GC-ECD were used.

In water treatment, chloramines, chlorine, ozone, and chlorine dioxide was used frequently as 
disinfectants to inactivate microorganisms and NOM. In this research, we choose chlorine as disinfectant. During the chlorination process, the existence of bromide also had a major effect on the distribution and formation of THMs [8].

The chlorination of AA in an actual water distribution systems (WDS) are affected by more factors than that in a beaker experiment, including NOM, pipe scales, pipe wall and so on [9].What's more, there is little report about the THMs in the presence of $\mathrm{Br}^{-}$in the WDS. Therefore, our experiments were performed on a simulative water distribution system (WDS) to analysis the potential of producing THMs. Simulative water distribution system has four loops, and each loop is approximately $80 \mathrm{~m}$ in length and $150 \mathrm{~mm}$ in diameter. Experimental water circulated in the loop came from the municipal drinking water network in Hangzhou City.

\section{Materials and methods}

Chemicals. Hexane and Methyl tert-butyl ether (MTBE) were chromatographic grade and purchased from Sigma-Aldrich. Trihalomethanes calibration mixtures were also purchased from Sigma-Aldrich. Sodium hypochlorite and Aspartate were analytical grade chemicals (Aladdin). The other chemicals such as Methanol, Potassium bromide were analytical grade (Sinopharm Chemical Reagent). All solutions were prepared with Ultra-pure water (Heal Force ultra-pure system) obtained from a Milli-Q system with resistivity $>18 \mathrm{M} \Omega \mathrm{cm}$.

Analytical procedures. Separation and identification of THMs were detected by gas chromatograph (Varian, GC-450), equipped with a splitless injector and a SP-Sil 5 DB capillary column $\left(30 \mathrm{~m} \times 0.25 \mathrm{~mm} \times 0.25 \mu \mathrm{m}\right.$, Varian). The oven was held at $35{ }^{\circ} \mathrm{C}$ for $10 \mathrm{~min}$, then was ramped at $10{ }^{\circ} \mathrm{C} / \mathrm{min}$ to $80{ }^{\circ} \mathrm{C}$, and ramped again to the final temperature of $150{ }^{\circ} \mathrm{C}$ at $20{ }^{\circ} \mathrm{C} / \mathrm{min}$ where it was held for $1 \mathrm{~min}$. The injector and detector temperatures were $210{ }^{\circ} \mathrm{C}$ and $290{ }^{\circ} \mathrm{C}$, respectively. Nitrogen was used as carrier gas with a flow rate of $1.5 \mathrm{~mL}$ per min. The detection of free residual chlorine were used DR2800 $(\mathrm{HACH})$.

Chlorination experiments. The curve was established by measuring ten samples of $\mathrm{CHCl}_{3}$, $\mathrm{CHBrCl}_{2}, \mathrm{CHBr}_{2} \mathrm{Cl}$ and $\mathrm{CHBr}_{3}$ at final concentration from 0 to $100 \mu \mathrm{g} / \mathrm{L}(0,0.2,0.5,1,2,5,10,20$, 50 and $100 \mu \mathrm{g} / \mathrm{L}$ ) using GC-ECD. Quantitative analysis was based on peak area measurements as ratios versus peak area of internal standard [10].All samples were treated as following sample preparation procedure. $20 \mathrm{~mL}$ ultrapure water samples were added to a $50 \mathrm{~mL}$ glass with $8 \mathrm{~g}$ of anhydrous sodium sulfate and $2 \mathrm{~mL}$ of MTBE (as extractant). Then $1 \mathrm{ml}$ supernatant was taken to GC-ECD after vortexing for sample homogenization. By fitting the calibration curve of four THMs, the regression coefficient was always 0.96 or better.

The experiments about amino acid used sodium hypochlorite as a disinfectant. The experiments were conducted at dark conditions under thermostat water bath in $100 \mathrm{~mL}$ airtight container. The initial dosage of aspartate was $0.1 \mathrm{mmol} / \mathrm{L}$. A chlorine stock solution was prepared by mixing $6 \%$ sodium hypochlorite and amino acid according the molar ratio. The stock solution was adjusted to a $\mathrm{pH}$ of 7.0 with hydrochloric acid or sodium hydroxide. The concentration of bromide ions in the stock solution was determined at $10 \mathrm{mg} / \mathrm{L} .1 \mathrm{~mL}$ samples were taken at $0,1,3,12,24,48,72 \mathrm{~h}$ and analyse by GC-ECD later.

THMs in chlorinated drinking water were tested in the WDS. Before the experiments, the water temperature and flow velocity in the WDS were adjusted using automated controls. The experiment conditions were stabilised at $\mathrm{pH}=7.3 \pm 0.3$, a flow velocity of $1.0 \mathrm{~m} / \mathrm{s}$, a water temperature of $25^{\circ} \mathrm{C} \pm 1{ }^{\circ} \mathrm{C}$. $5 \%$ sodium hypochlorite was added to three different pipes. The amount of Asp is 
determined by the ratio of chlorine concentration after the reaction of 150 minutes in the pipe. During the circulation of water in the WDS, $1 \mathrm{~L}$ samples were taken at $0 \mathrm{~h}, 24 \mathrm{~h}, 48 \mathrm{~h}, 72 \mathrm{~h}$, then these samples were added $2 \mathrm{~mL}$ of a $100 \mathrm{mg} / \mathrm{L}$ sodium thiosulfate solution to stop the further reaction.

\section{Results and discussion}

THMs formation potential in beaker experiment. The bromide ion is oxidized by chlorine to hypobromous acid or hypobromous ion, which in turn reacts with NOM, forming brominated THMs $[3,11]$. The influence of some water quality parameters was carried out under different disinfection conditions, including free residual chlorine and bromide concentration.

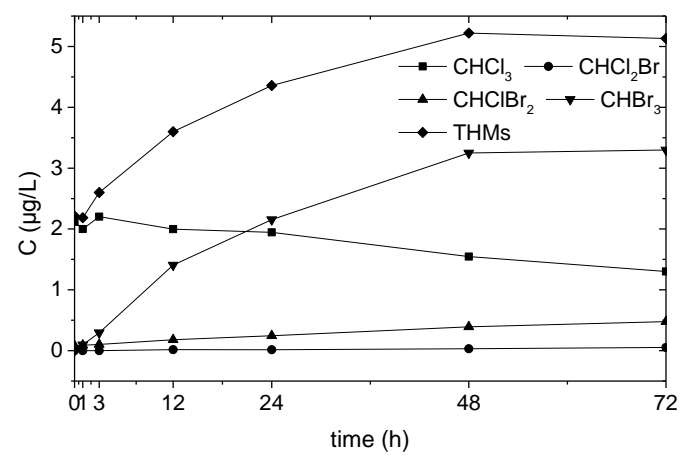

Fig1 the results of THMs in the beaker experiments $\left(\mathrm{n}_{\mathrm{c} 22}: \mathrm{n}_{\mathrm{Asp}}=10: 1, \mathrm{Br}=10 \mathrm{mg} / \mathrm{L}, \mathrm{pH}=7.0, \mathrm{~T}=20^{\circ} \mathrm{C}\right)$

When there was certain bromine ions existed in water, distribution and productions of THMs after Asp chlorination could be obtained from Figure 1. First of all, the generation amount of THMs gradually increased along with time. As the Asp chlorination proceeding, more THMs would be formed, but after 48 hours, the chlorination had stopped, and generation amount of THMs was unchanged. Secondly, generation amount of CF decreased along with the reaction, which was due to the presence of bromide ions, more chloroform translated into other THMs. Thirdly, generation amount of BDCM and DBCM proportionally increased follow the reaction proceeding, which was formed by $\mathrm{Br}$ - reacting with $\mathrm{CF}$. What's more, generation amount of $\mathrm{BF}$ also increased, and generation rate of $\mathrm{BF}$ was fast at the beginning of reaction, since the concentration of bromide ions was large. After 48 hours, generation rate of BF slow down with the concentration of bromide ions decreased.

In a word, Asp chlorination generated four kinds of THMs with the presence of bromide ions. Generation amount of each THMs were: $\mathrm{BF}>\mathrm{CF}>\mathrm{DBCM}>\mathrm{BDCM}$. When concentrations of bromide ions were larger, effect of bromine substitution is stronger, BF formed faster. Conversely, effect of bromine substitution was smaller at low concentrations of bromine ions, so BDCM and DBCM generated more.

THMs formation potential in the WDS. The water was tested before the chlorination experiments, and the conventional index in the stable WDS was showed in table 1. When treated water run for a long time in the WDS, the water stability of Loop B (the material was PE) was better than Loop C (the material was stainless steel) and Loop D (the material was ductileiron). 
Table 1 conventional index of experiments in the WDS

\begin{tabular}{cccc}
\hline & Loop B & Loop C & Loop D \\
\hline $\mathrm{pH}$ & 7.11 & 7.40 & 7.63 \\
$\mathrm{DO}(\mathrm{mg} / \mathrm{L})$ & 8.81 & 9.03 & 8.25 \\
Conductivity $(\mu \mathrm{S} / \mathrm{cm})$ & 297.3 & 241.3 & 293.8 \\
Turbidity $(\mathrm{NTU})$ & 1.06 & 2.08 & 0.84 \\
\hline
\end{tabular}

The formation of THMs is obvious different by comparing water samples from the asp chlorination and the water samples without asp chlorination. Figure 3 illustrates the formation of THMs as a function of chlorine contact time with bromide in the different pipe walls.
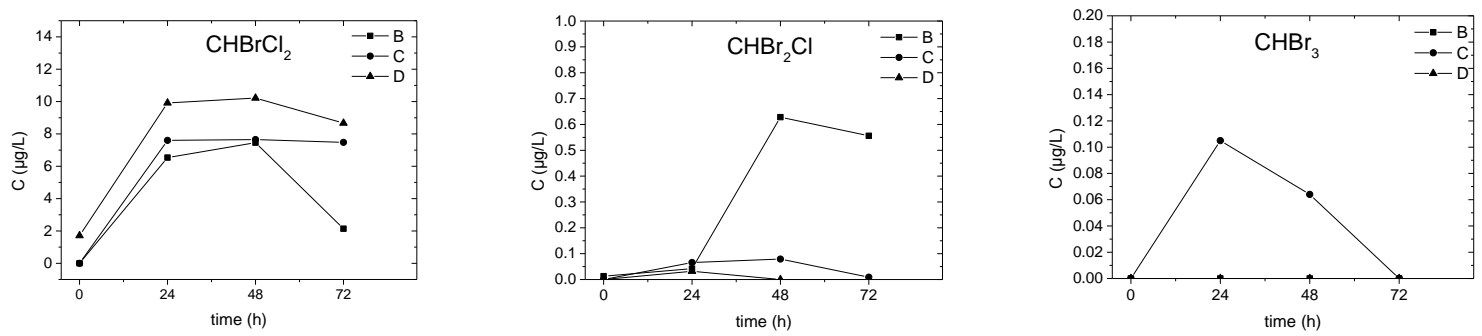

Fig. 2 Effect of bromide on the potential of THMs (the material of B was PE, C was stainless steel, D was ductileiron, and reaction conditions: $\left.\mathrm{n}_{\mathrm{cl} 2}: \mathrm{n}_{\mathrm{Asp}}=10: 1, \mathrm{Br}^{-}=10 \mathrm{mg} / \mathrm{L}, \mathrm{pH}=7.0, \mathrm{~T}=20^{\circ} \mathrm{C}\right)$

As can be seen in Fig.2, BDCM were existed most in all three different pipes (the material of B was PE, $\mathrm{C}$ was stainless steel, and $\mathrm{D}$ was ductileiron). Besides, the concentrations of $\mathrm{BF}$ and DBCM were lower than $1 \mu \mathrm{g} / \mathrm{L}$, and no CF was existed. Since the oxidation of $\mathrm{HOBr}$ is more powerful than $\mathrm{HOCl}$, the $\mathrm{CF}$ could be easily oxidized to the BDCM when the bromide ion was presented in small amounts. In addition, there were no enough bromide ions in the pipe to oxidizing all of chlorides. Therefore, we can use BDCM concentration to replace the THMs.

In fig.2 (a), with the increase of the chlorination time, the concentration of BDCM was also increasing rapidly in the first 24 reaction hours. Then the ratio of producing BDCM was slow down since free chlorine and bromide consumed. The difference becomes significant in the final stage. Microorganisms on the pipe wall which attached growth began to decompose after 48 hours of chlorination reaction, thus, the concentration of BDCM reduced respectively at $2.138 \mu \mathrm{g} / \mathrm{L}, 7.477$ $\mu \mathrm{g} / \mathrm{L}$ and $8.67 \mu \mathrm{g} / \mathrm{L}$. However, formation potential of THMs was not the same in different pipe walls. From fig 3, the generation potential of THMs in three different pipe walls was: the material of $\mathrm{PE}>$ stainless steel > ductileiron. This is apparently related to trace microorganisms and chemical elements on the wall of pipes, which is an important factor that affects the reaction rate in the drinking water. In brief, the characteristic of pipe is more unstable, and the THMs will be generated more easily.

Fig. 3 showed that generation amount of THMs in the loop D was always the most. In addition, the presence of THMs is the most stable in loop $\mathrm{C}$ with the minimal changing. So it is recommended that choosing $\mathrm{PE}$ pipe as drinking water conveyance is healthier. 


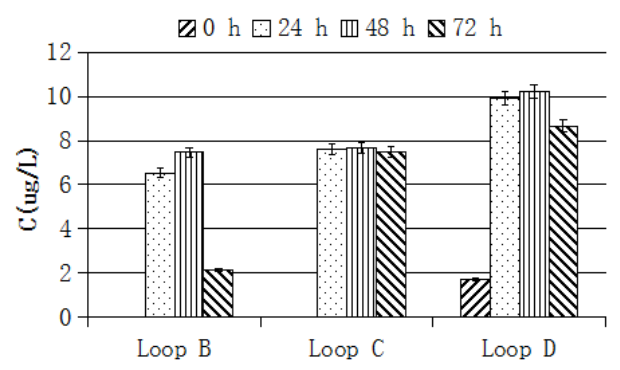

Fig. 3 the potential of $\mathrm{CHBrCl} 2$ in different pipes

The Experiment in beaker contrast with that in WDS with the same settings, and it showed a big difference in experiment results. The generation amount of BF in Beaker experiment is the most one, but the generation amount of BF in WDS is almost zero. This phenomenon indicates that bromide ions in WDS are easily decomposed by microorganisms to be not replaced several times. Since the pipe environment is more complex, the reaction time of beaker experiment is significantly faster than the WDS experiment. Moreover, the generation amount of THMs in WDS is less than in beaker, so the pipe environment has an absorption and decomposition effects for THMs.

\section{Conclusions}

Most studies on the formation and control of THMs have focused on drinking water treatment utilities using surface water sources, wastewater treatment systems, and power plants, while limited research has focused on THMs in pilot-scale WDS. The generation amount of each THMs in beaker experiment are: $\mathrm{BF}>\mathrm{CF}>\mathrm{DBCM}>\mathrm{BDCM}$. And the generation potential of THMs in three different pipe walls was: the material of $\mathrm{PE}>$ stainless steel $>$ ductileiron. What's more, the reaction time of beaker experiment is significantly slower than the WDS experiment. The presence of bromide ions can affect the generation of THMs, and the pipe environment will also reduce the generation of THMs. From a security point of view, we recommend using PE pipe in water distribution systems.

\section{Acknowledgments}

This work was kindly supported by National Natural Science Foundation of China (No. 51208455,5157080749), the National Major Program on Pollution Control and Management of Water Body (Grant No. 2012ZX07403-003), Zhejiang Provincial Innovative Research Team (Y15E080016).

\section{References}

[1] J. J. Rook. Environmental Science \& Technology, 1977, 11(5): 478-482.

[2] L Liang, P C Singer. Environmental Science \& Technology, 2003, 37(13): 2920-2928.

[3] J Hu, H Song and T Karanfil. Environmental science \& technology, 2009, 44(2): 794-799.

[4] A Li, X Zhao, R Mao, et al. Journal of Hazardous Materials, 2014. 271: p. 228-235.

[5] M J Nieuwenhuijsen, J Grellier, R Smith, et al. Philosophical Transactions of the Royal Society of London A: Mathematical, Physical and Engineering Sciences, 2009, 367(1904): 4043-4076.

[6] A Sun, W Chen, T Lin, et al. CLEAN-Soil, Air, Water, 2010, 38(4): 353-360.

[7] H Hong, Y Xiong, M Ruan, et al. Science of The Total Environment, 2013, 444: 196-204. 
[8] G Hua, D A Reckhow. Environmental science \& technology, 2007, 41(9): 3309-3315.

[9] C Li, Z Wang, Y J Yang, et al. Chemosphere, 2015, 125: 86-93.

[10] P Charisiadis, K. C Makris. Journal of Chromatography B, 947, 17-22.

[11] S. W. Krasner, H. S.Weinberg, S. D. Richardson, et al. Environmental science \& technology, 40 (23), 7175-7185. 